\title{
Erratum to: Evaluation of bleeding on probing and gingival crevicular fluid enzyme activity for detection of periodontally active sites during supportive periodontal therapy
}

\author{
Hiroshi Ito • Yukihiro Numabe - Satoshi Sekino • Etsuko Murakashi • Hitomi Iguchi - Shuichi Hashimoto •

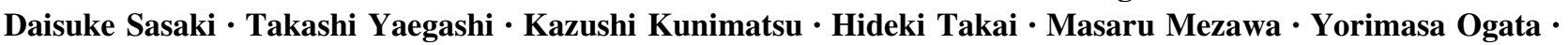 \\ Hisashi Watanabe $\cdot$ Satsuki Hagiwara $\cdot$ Yuichi Izumi $\cdot$ Yuka Hiroshima $\cdot$ Jun-Ichi Kido $\cdot$ Toshihiko Nagata
}

Published online: 16 January 2013

(C) The Society of The Nippon Dental University 2013

\section{Erratum to: Odontology}

DOI 10.1007/s10266-012-0090-1

The authors would like to correct certain errors in the original publication of the article.

In Table 3 the letters in the columns were inadvertently misaligned and are corrected as given and in Table 5 $\mathrm{BOP}(-) n=14$ was incorrectly published as $\mathrm{OP}(-)$ $n=14$.

The online version of the original article can be found under doi:10.1007/s10266-012-0090-1.

H. Ito $(\square) \cdot$ Y. Numabe $\cdot$ S. Sekino · E. Murakashi · H. Iguchi Department of Periodontology, The Nippon Dental University School of Life Dentistry at Tokyo, 1-9-20 Fujimi, Chiyoda-ku, Tokyo 102-8159, Japan

e-mail: hiroshi@tky.ndu.ac.jp

\section{S. Hashimoto}

Dental Research Institute, Radio Isotope Center, The Nippon Dental University School of Life Dentistry at Tokyo,

1-9-20 Fujimi, Chiyoda-ku, Tokyo 102-8159, Japan

\section{Sasaki · T. Yaegashi · K. Kunimatsu}

Division of Periodontology, Department of Conservative Dentistry and Oral Rehabilitation, Iwate Medical University School of Dentistry, 1-3-27 Chuo-dori, Morioka,

Iwate 020-0085, Japan

H. Takai · M. Mezawa · Y. Ogata

Department of Periodontology, Nihon University School

of Dentistry at Matsudo, 2-870-1 Sakaecho-Nishi,

Matsudo, Chiba 271-8587, Japan
H. Watanabe $\cdot$ S. Hagiwara $\cdot$ Y. Izumi

Section of Periodontology, Department of Hard Tissue

Engineering, Graduate School of Medical and Dental Science,

Tokyo Medical and Dental University, 1-5-45 Yushima,

Bunkyo-ku, Tokyo 113-8549, Japan

Y. Hiroshima $\cdot$ J.-I. Kido $\cdot$ T. Nagata

Department of Periodontology and Endodontology, Institute of Health Biosciences, The University of Tokushima Graduate School, 3-18-15 Kuramoto, Tokushima 777-8504, Japan 
Table 3 Correlation with clinical parameters and biochemical data in each category divided by BOP and PPD

\begin{tabular}{|c|c|c|c|c|c|c|c|c|c|c|c|c|}
\hline & \multicolumn{3}{|c|}{$\begin{array}{l}\text { BOP }(-) \text { group with PPD } \\
\leq 4 \mathrm{~mm}(205 \text { sites })\end{array}$} & \multicolumn{3}{|c|}{$\begin{array}{l}\text { BOP }(+) \text { group with PPD } \\
\leq 4 \mathrm{~mm}(22 \text { sites })\end{array}$} & \multicolumn{3}{|c|}{$\begin{array}{l}\text { BOP }(-) \text { group with PPD } \\
\geq 5 \mathrm{~mm} \text { (96 sites) }\end{array}$} & \multicolumn{3}{|c|}{$\begin{array}{l}\text { BOP }(+) \text { group with PPD } \\
\geq 5 \mathrm{~mm}(78 \text { sites })\end{array}$} \\
\hline & $\begin{array}{l}\mathrm{NE} \\
\text { activity }\end{array}$ & $\begin{array}{l}\text { AST } \\
\text { activity }\end{array}$ & $\begin{array}{l}\text { Amount } \\
\text { of protein }\end{array}$ & $\begin{array}{l}\mathrm{NE} \\
\text { activity }\end{array}$ & $\begin{array}{l}\text { AST } \\
\text { activity }\end{array}$ & $\begin{array}{l}\text { Amount } \\
\text { of protein }\end{array}$ & $\begin{array}{l}\mathrm{NE} \\
\text { activity }\end{array}$ & $\begin{array}{l}\text { AST } \\
\text { activity }\end{array}$ & $\begin{array}{l}\text { Amount } \\
\text { of protein }\end{array}$ & $\begin{array}{l}\mathrm{NE} \\
\text { activity }\end{array}$ & $\begin{array}{l}\text { AST } \\
\text { activity }\end{array}$ & $\begin{array}{l}\text { Amount } \\
\text { of protein }\end{array}$ \\
\hline \multicolumn{13}{|l|}{ PII } \\
\hline $\mathrm{CC}$ & 0.023 & 0.283 & 0.163 & 0.141 & 0.233 & 0.131 & -0.181 & 0.244 & -0.085 & 0.091 & 0.099 & 0.084 \\
\hline SP & 0.742 & $0.000 * *$ & $0.019 *$ & 0.532 & 0.296 & 0.561 & 0.078 & $0.017 *$ & 0.410 & 0.426 & 0.388 & 0.463 \\
\hline \multicolumn{13}{|l|}{ GI } \\
\hline $\mathrm{CC}$ & -0.058 & 0.030 & 0.107 & 0.278 & -0.121 & 0.260 & 0.031 & 0.205 & 0.193 & 0.071 & 0.204 & 0.023 \\
\hline SP & 0.411 & 0.672 & 0.128 & 0.210 & 0.593 & 0.243 & 0.763 & $0.045^{*}$ & 0.059 & 0.538 & 0.073 & 0.841 \\
\hline \multicolumn{13}{|l|}{ CAL } \\
\hline $\mathrm{CC}$ & 0.010 & 0.379 & 0.192 & -0.138 & -0.400 & -0.126 & -0.010 & 0.012 & -0.002 & 0.082 & 0.027 & 0.045 \\
\hline SP & 0.888 & $0.000 * *$ & $0.006^{* *}$ & 0.541 & 0.065 & 0.577 & 0.925 & 0.904 & 0.981 & 0.473 & 0.817 & 0.695 \\
\hline \multicolumn{13}{|l|}{ PPD } \\
\hline $\mathrm{CC}$ & -0.033 & 0.318 & 0.204 & 0.210 & -0.007 & 0.411 & 0.316 & 0.088 & 0.167 & 0.068 & 0.213 & 0.106 \\
\hline SP & 0.636 & $0.000 * *$ & $0.003 * *$ & 0.349 & 0.977 & 0.058 & $0.002 * *$ & 0.395 & 0.105 & 0.557 & 0.061 & 0.356 \\
\hline \multicolumn{13}{|l|}{ GCF } \\
\hline $\mathrm{CC}$ & 0.210 & 0.362 & 0.448 & 0.369 & 0.508 & 0.666 & 0.096 & 0.438 & 0.464 & 0.501 & 0.092 & 0.460 \\
\hline SP & $0.003 * *$ & $0.000 * *$ & $0.000 * *$ & 0.091 & $0.016^{*}$ & $0.001 * *$ & 0.350 & $0.000 * *$ & $0.000 * *$ & $0.000 * *$ & 0.425 & $0.000 * *$ \\
\hline
\end{tabular}

$C C$ coefficient of correlation, $S P$ significant probability

* Significant: $p<0.05$

** Significant: $p<0.01$ 